\title{
Overexpressed Derlin-1 Inhibits ER Expansion in the Endothelial Cells Derived from Human Hepatic Cavernous Hemangioma
}

\author{
Dong Hu${ }^{1}$, Yu-Liang Ran ${ }^{1}$, Xing Zhong ${ }^{1}$, Hai Hu${ }^{1}$, Long Yu${ }^{1}$, Jin-Ning Lou ${ }^{2}$, Li-Xing Sun ${ }^{1}$ and Zhi-Hua Yang,* \\ ${ }^{1}$ Department of Cell and Molecular Biology, Cancer Institute and Cancer Hospital, \\ Chinese Academy of Medical Sciences and Peking Union Medical College, Beijing, 100021, P.R. China \\ ${ }^{2}$ Institute of Clinical Medical Sciences, China-Japan Friendship Hospital, Beijing 100029, P.R. China
}

Received 2 December 2005, Accepted 28 June 2006

\begin{abstract}
Proteins that are unfolded or misfolded in the endoplasmic reticulum (ER) must be targeted for refolding or degradation to maintain the homeostasis of the ER. Derlin-1 was reportedly implicated in the retro-translocation of misfolded proteins from the ER to the cytosol for degradation. In this report, we showed that Derlin-1 was down-regulated in the endothelial cells derived from human hepatic cavernous hemangioma (CHEC) compared with other tested cells. Electron microscopy analysis showed that ER was aberrantly enlarged in CHEC cells, but not in other tested cells. When overexpressed, Derlin-1 induced the dilated ER to return normal size. This ER dynamic was associated with the activation of unfolded protein response (UPR). In CHEC cells where Derlin-1 was down-regulated, increased expression of the immunoglobulin heavy chain-binding protein (Bip) and UPR-specific splicing of X-box DNAbinding protein 1 (XBP1) mRNA were detected, as compared with that in other tested cells, indicating that UPR was activated. After Derlin-1 overexpression, the extent of UPR activation diminished, as evidenced by decreased expression of Bip, reduced amount of the spliced form of XBP1 (XBP1s), and elevated expression of the unspliced form of XBP1 (XBP1 $\left.1_{U}\right)$. Taken together, these findings provide another example of a single protein being able to affect ER dynamic in mammalian cells, and an insight into the possible molecular mechanism(s).
\end{abstract}

Keywords: Bip, Derlin-1, Endoplasmic reticulum, Unfolded protein response, $\mathrm{XBP} 1$

*To whom correspondence should be addressed.

Tel: 86-10-87771740; Fax: 86-10-67783169

E-mail: yang_zhihua_prof@yahoo.com.cn

\section{Introduction}

Endoplasmic reticulum (ER) is a multifaceted organelle. It functions primarily to facilitate the folding and assembly of newly synthesized secretory and membrane proteins. Although ER provides the optimal environment for protein folding and assembly, the large flux of proteins through the ER would inevitably bring about a consequence that the folding and assembly process fails, resulting in the production of unfolded or misfolded proteins. To cope with this problem, eukaryotic cells have developed two distinct processes, namely unfolded protein response (UPR) and ER-associated degradation (ERAD). ERAD and UPR are tightly linked processes. Either cellular stress or loss of ERAD would result in accumulation of misfolded proteins and thereby activation of the UPR, whereas UPR acts to reduce levels of misfolded proteins by inhibiting the formation of misfolded species, increasing the folding capacity, and enhancing the rate of ERAD (Travers et al., 2000).

UPR is a complex signal-transduction cascade, responsible for dealing with excessive accumulation of unfolded or misfolded proteins in the ER (Schroder and Kaufman, 2005). When activated, UPR acts to limit new protein synthesis, increase expression of certain genes involved in expanding the folding capacity of ER, and accelerate the degradation of misfolded proteins. To date, two UPR specific pathways have been identified. One branch is directed by inositol-requiring gene 1 (IRE1), an ER membrane-bound endoribonuclease (Mori, 2003; Ma and Hendershot, 2004). When activated, IRE1 removes 26 bases from XBP1 transcript, generating spliced XBP1 $\left(\mathrm{XBP} 1_{\mathrm{S}}\right)$ mRNA, which encodes a transcription factor capable of activating transcription efficiently. Another branch of the UPR is mediated by activating transcription factor 6 (ATF6), an ER membrane-bound transcription factor. Upon ER stress, ATF6 translocates to the Golgi apparatus, sequentially cleaved by site 1 and site 2 proteases, liberating the cytosolic transcription factor domain, which translocates 
to the nucleus and activates transcription (Mori, 2003; Yamamoto et al., 2004).

ERAD is a process by which misfolded proteins are recognized and directed to the translocon for retrotranslocation from the ER to the cytosol, where they are degraded in an ubiquitin- and proteasome-dependent manner (Shen et al., 2004). Attempts to characterize the ERAD process have identified many components of ERAD machinery, including Sec61p (Kalies et al., 2005) and ER degradation enhancing $\alpha$-mannosidase-like protein (EDEM) (Hosokawa et al., 2001). Recently, two groups have independently identified a novel component of ERAD Derlin1 (Lilley and Ploegh, 2004; Ye et al., 2004). Derlin-1 is considered to provide the missing link between events on the luminal side of the ER (recognition of misfolded protein substrates) and those on the cytosolic side (ubiquitination catalyzed by ubiquitin ligases and extraction catalyzed by p97 ATPase) (Oda et al. 2006). During retro-translocation, Derlin1 may first receive misfolded protein substrates from ER chaperones (Tsai et al., 2002). Next, the substrates are translocated across the membrane through a proteinconducting channel that is postulated to be formed by Derlin1 homo-oligomers (Ye et al., 2005). Once the substrates have emerged into the cytosol, a membrane complex containing p97, VCP (another name for p97)-interacting membrane protein (VIMP) and ubiquitin ligases is recruited to substratebound Derlin-1 by VIMP. Ubiquitin ligases catalyze polyubiquitination of the substrates, whereas p97 uses ATP hydrolysis to pull the ubiquitinated substrates across the ER membrane to the cytosol for degradation by the proteasome (Ye et al., 2004; Ye et al., 2005). With regard to the misfolded glycoprotein substrates, their degradation by the proteasome after retro-translocation requires an additional process deglycosylation. Peptide:N-glycanase (PNGase) has been recently reported to associate with Derlin-1, thereby bringing it in close proximity to the ER membrane and providing accessibility to dislocating glycoproteins, which may be required for the deglycosylation of a subset of misfolded glycoprotein substrates, such as MHC class I heavy chains (HCs) (Katiyar et al., 2005).

Derlin-1 homologues in other species have been identified, including R151.6 in Caenorhabditis elegans (Hough et al., 1999), Zm (Zea mays) Derlin1-1 (Kirst et al., 2005), At (Arabidopsis thaliana) Derlin1-1 and Der1 in Saccharomyces cerevisiae (Knop et al., 1996). Their functions are well conserved. It was reported that the ER was considerably enlarged in $\Delta$ derl yeast cells, whereas a $\Delta$ derl 1 irel double knockout strain had no proliferated ER, suggesting that UPR plays a critical role in the ER dynamic affected by the der 1 mutation (Taxis et al., 2002). Previously, we had shown that Derlin-1 was ubiquitously expressed in various kinds of endothelial cells, but markedly down-regulated in the endothelial cells derived from human hepatic cavernous hemangioma (CHEC) (unpublished data). Electron microscopy analysis showed that ER was considerably expanded in
CHEC cells, but not in other tested cells. The relation between Derlin-1 and ER dynamic in mammalian cells remains to date unreported. In this study, we demonstrate that overexpressed Derlin-1 inhibits ER expansion in CHEC cells, and suggest that UPR may be one of the mechanisms underlying the ER expansion.

\section{Materials and Methods}

Cell culture. Human liver sinusoidal endothelial cells (LSEC) were established and maintained as previously described (Salmon et al., 2000). The endothelial cells derived from human hepatic cavernous hemangioma (CHEC) were isolated and purified by Dynabeads (Dynal A.S.) coated with Ulex europaeus agglutinin-1 (UEA-1) (Lou et al., 1998; Lou et al., 1999). Several endothelial cell markers, such as von Willebrand factor (vWF), CD31, and CD34, were used to identify the purified CHEC cells by immunofluorescence staining. The purity of CHEC cells was confirmed by fluorescenceactivated cell sorting (FACS) analysis for vWF expression. Human umbilical vein endothelial cells (HUVEC) were isolated and cultured as described (Jaffe et al., 1973). Human liver cancer endothelial cells (LCEC) and human esophagus carcinoma endothelial cells (ECEC) were isolation and identified according to the previously method (Zhong et al., 2004). All these kinds of endothelial cells, except immortalized LSEC cells, were used between 6-9 passages in this study.

RNA isolation and RT-PCR. Total RNA was isolated from cells using TRIzol reagent (Invitrogen) according to the manufacturer's manual. Two micrograms of total RNA were subjected to reverse transcription for $60 \mathrm{~min}$ at $42^{\circ} \mathrm{C}$ using 200U of Moloney murine leukemia virus (M-MLV) reverse transcriptase (Promega).

Semi-quantitative RT-PCR was performed in a $25 \mu$ l reaction mixture including an aliquot corresponding to $1 / 25$ of the resulting cDNA. The PCR program for amplification was as follows: initial denaturation at $94^{\circ} \mathrm{C}$ for $3 \mathrm{~min}, 25-28$ cycles at $94^{\circ} \mathrm{C}$ for $30 \mathrm{~s}, 55^{\circ} \mathrm{C}$ for $30 \mathrm{~s}, 72^{\circ} \mathrm{C}$ for $30 \mathrm{~s}$, and a final incubation at $72^{\circ} \mathrm{C}$ for $10 \mathrm{~min}$. PCR products were separated on a $2 \%$ agarose gel. Internal control RT-PCR of $\beta$-actin was done on all specimens simultaneously. The PCR primers used span different exons of corresponding genes. Their sequences are as follows: Derlin-1 (forward [5'-TCG GCA AAC TCG GCC TCA TC-3'] and reverse [5'-TCT CCC GCC TCC GCC ATT CT-3']), $\beta$-actin (forward [5'-GGC GGC ACC ACC ATG TAC CCT-3'] and reverse [5'-AGG GGC CGG ACT CGT CAT ACT-3']).

To analysis the splicing of XBP1 mRNA, RT-PCR was performed with forward (5'-CCT TGT AGT TGA GAA CCA GG-3') and reverse (5'-GGG GCT TGG TAT ATA TGT GG-3') primers following the same procedure as above. 442 and $416 \mathrm{bp}$ fragments, which represent spliced $\left(\mathrm{XBP} 1_{\mathrm{S}}\right)$ and unspliced $\left(\mathrm{XBP} 1_{\mathrm{U}}\right) \mathrm{XBP} 1$ respectively, were documented after separation on a $2 \%$ agarose gel (Shang and Lehrman, 2004).

Antibody preparation and western blot analysis of Derlin-1. Anti-Derlin-1 antisera were generated in New Zealand White rabbits against peptides coupled to keyhole-limpet haemocyanin (KLH). 
The sequence of used peptides was (C) RHNWGQGFRLGDQ. The initial immunization was done in complete Freund's adjuvant (Sigma Chemical Co.) and subsequent boosters were with imcomplete Freund's adjuvant (Sigma Chemical Co.). The antibody specific to Derlin-1 was affinity purified on sepharose gel coupled with the used peptides.

Cells were washed with PBS and lysed in SDS sample buffer. After brief sonication and boiling, proteins were separated on a $12 \%$ SDS-PAGE and transferred to the $0.2 \mu \mathrm{m}$ Immobilon PVDF membranes (Millipore, Billerica). Membranes were incubated in a block solution (5\% skimmed milk, $0.1 \%$ Tween-20 in PBS) for $60 \mathrm{~min}$, washed with PBST (PBS with $0.1 \%$ Tween-20), and probed with primary antibodies diluted in the block solution (antiDerlin-1, $1 \mu \mathrm{g} / \mathrm{ml}$; anti-Bip, $1: 500$, Santa Cruz Biotechnology, $\beta$ actin, $1: 5,000$, Sigma Chemical Co.) at $4^{\circ} \mathrm{C}$ overnight. Anti-human $\beta$-actin antibody was used as the internal control. Following washing and incubation with horseradish peroxidase (HRP)conjugated secondary antibody (Zhongshan Company) at $1: 5000$ in the block solution for $60 \mathrm{~min}$, membranes were washed, detected by ECL (Amersham), and visualized by exposure to X-ray film (Amersham).

Electron microscopy. BD Falcon Cell Culture Inserts (Becton, Dickinson and Company) were coated with $100 \mu \mathrm{l}$ of $20 \mathrm{~g} / \mathrm{l}$ gelatin (Sigma Chemical Co.) at $4^{\circ} \mathrm{C}$ overnight in a humidified chamber. The coated inserts were incubated at $37^{\circ} \mathrm{C}$ for $30 \mathrm{~min}$ and cells were plated, cultured overnight. The membranes of the inserts with cells growing on them were washed with PBS, fixed in $2 \%$ glutaraldehyde, and then in $1 \%$ osmic acid. Following dehydration by sequential extraction with graded concentrations of ethanol and clearing process with propylene oxide, the membranes were embedded in Epon 812 resin, sectioned on an ultramicrotome at high speed. The resulting thin sections were mounted on nickel grids, stained with $1 \%$ uranyl acetate (prepared fresh) and Reynolds' lead citrate, and assessed using a Hitachi 7100 electron microscope.

DNA construction and transfection. The full-length of Derlin-1 cDNA was amplified with the following primers: 5'-ATT CGC GGA TCC ATG TCG GAC ATC GGA GAC TGG T-3' and 5'TAG CCG GAA TTC TCA CTG GTC TCC AAG TCG AAA G3'. The restriction sites were included (underlined) to facilitate the cDNA product cloned into pcDNA3 (Invitrogen).

CHEC cells were transfected with Derlin-1-pcDNA3 or pcDNA3 control as described (Dalby et al. 2004) with some modifications. Briefly, $1 \times 10^{5}$ cells were plated and grown to be at least $80 \%$ confluent on the next day for transfection. $4 \mu \mathrm{g}$ plasmid and $2.5 \mu \mathrm{l}$ Lipofectamine 2000 (Invitrogen) were diluted in $250 \mu$ Opti-MEM I reduced serum medium (Invitrogen) respectively, mixed, and added to each well. Following incubation for $4 \mathrm{~h}$, the transfection mixture was replaced by fresh medium. The six-well plates were cultured for $48 \mathrm{~h}$ and subjected to subsequent analysis.

Statistical analysis. The Student's test was used to evaluate the significance of differences between groups. The level of significance was set at $p<0.05$.

\section{Results}

Down-regulation of Derlin-1 in CHEC cells. In a previous effort to isolate tumor angiogenesis-associated genes, we have identified Derlin-1 as one of the genes overexpressed in tumor endothelial cells (unpublished data). To further confirm the expression of Derlin-1 in various kinds of endothelial cells, both RT-PCR and Western blot were used. The tested endothelial cells derived from human umbilical vein (HUVEC), human liver sinus (LSEC), human hepatic cavernous hemangioma (CHEC), human liver cancer (LCEC), and human esophagus carcinoma (ECEC), respectively. Derlin-1 mRNA and protein level were measured by densitometric analysis of RT-PCR (Fig. 1A) and Western blot (Fig. 1C) respectively. Quantitative evaluation of the relative expression of Derlin-1 mRNA (Fig. 1B) and protein (Fig. 1D) revealed that Derlin-1 was downregulated by approximate $70 \%$, even larger scale, in CHEC cells compared with other tested cells.

Recently, two additional Der1p-homologous proteins, designated Derlin-2 and -3, have been identified (Lilley and Ploegh, 2005; Oda et al., 2006). Similar to Derlin-1, they are both suggested to be required for ERAD in mammalian cells as well (Oda et al., 2006). Amino acid sequence alignment of human Derlin-1, Derlin-2, and Derlin-3 indicated that the sequence of peptides used for Derlin-1 antibody preparation showed no homology to Derlin-2 and -3. Therefore, Derlin-1 antibody used did not react to Derlin-2 and -3 , which was further confirmed in the subsequent study, where Derlin-1 was up-regulated after introduction of Derlin-1 construct into CHEC cells.

Overexpressed Derlin-1 inhibited ER expansion in CHEC cells. Derlin-1 has previously been implicated in disposal of misfolded proteins from the ER to the cytosol (Lilley and Ploegh, 2004; Ye et al., 2004; Lilley and Ploegh, 2005; Ye et al., 2005). Down-regulation of Derlin-1 might result in excessive accumulation of misfolded proteins in ER, and thus affect ER dynamic. It has been reported that ER is considerably enlarged in $\Delta d e r l$ yeast cells (Taxis et al., 2002). Therefore, transmission electron microscopy (TEM) was used to examine the ER ultrastructure. Three kinds of endothelial cells were used, including CHEC, LCEC, and LSEC cells. For each cell line, at least fifty cells were analyzed. Compared with LCEC and LSEC cells, CHEC cells contained enlarged rough ER (RER), which was characterized by intracellular membrane-bound structures that were frequently studded with ribosomes (Fig. 3A).

To further determine whether Derlin-1 plays a role in the ER expansion in CHEC cells, Derlin-1 was overexpressed and ER expansion was examined. We first amplified the fulllength of Derlin-1 cDNA and cloned it into the eukaryotic expression vector pcDNA3, generating Derlin-1-pcDNA3. CHEC cells were transfected with Derlin-1-pcDNA3 and 
(A)

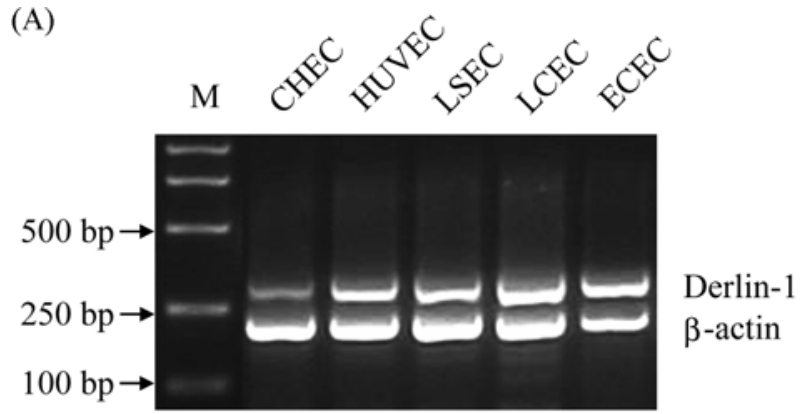

(C)

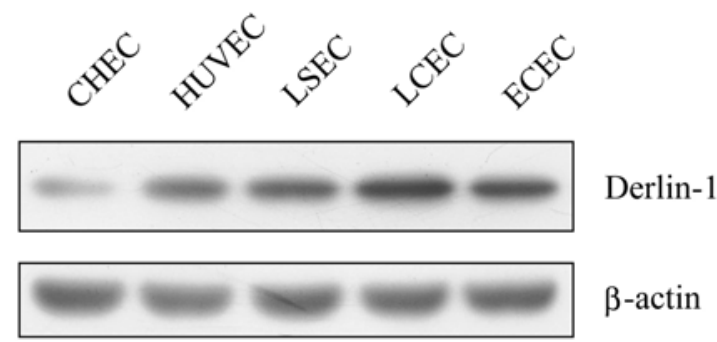

(B)

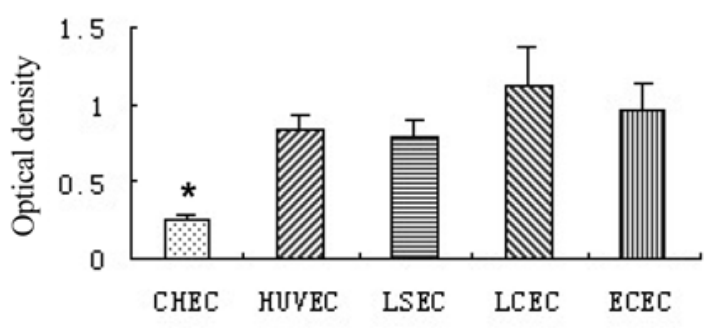

(D)

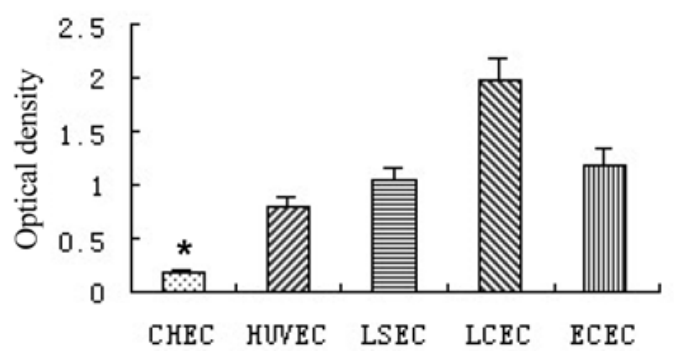

Fig. 1. Derlin-1 expression in endothelial cells. (A) RT-PCR analysis of Derlin-1 mRNA expression in the indicated endothelial cells. $\beta$ actin served as the internal control, and was amplified with Derlin-1 in the same reaction. M, DL2, 000 DNA marker. (B) Derlin-1 mRNA level quantitated from RT-PCR in (A). The absorbance of the bands was measured by densitomeric analysis. The relative expression of Derlin-1 mRNA was quantitated from three separate experiments and normalized to that of $\beta$-actin. Columns, mean; bars, SE (CHEC cells versus other tested cells, ${ }^{*} p<0.05$ ). (C) Western blot analysis of Derlin-1 protein expression in the indicated cells. (D) Derlin-1 protein level quantitated from Western blot in (C). Columns, mean for three separate experiments; bars, SE (CHEC cells versus other tested cells, ${ }^{*} p<0.05$ ). CHEC, endothelial cells derived from human hepatic cavernous hemangioma; HUVEC, human umbilical vein endothelial cells; LSEC, human liver sinus endothelial cells; LCEC, human liver cancer endothelial cells; ECEC, human esophageal cancer endothelial cells.

empty vector pcDNA3, respectively. Combination of RT-PCR and Western blot analysis showed that the expression level of Derlin-1 mRNA and protein were both significantly upregulated, an approximate 3- to 4-fold increase in Derlin-1pcDNA3-transfected cells compared with mock or empty vector-transfected control, thus confirming the efficacy of transfection (Fig. 2). Next, ER ultrastructure was examined. Fifty cells per sample were analyzed. The enlarged ER was diminished in $44 \%$ to $64 \%$ of Derlin-1-pcDNA3-transfected CHEC cells, whereas no changes were detected in all analyzed empty vector-transfected CHEC cells (Fig. 3B).

UPR activation diminished with Derlin-1 overexpression. UPR has been suggested to play an important role in regulation of ER biogenesis (Shaffer et al., 2004; Sriburi et al., 2004; Federovitch et al., 2005). In yeast cells, UPR is essential for ER proliferation induced by der 1 deletion mutation (Taxis et al., 2002). To determine the possible molecular mechanism(s) responsible for the ER dynamic affected by Derlin-1 overexpression, UPR activation was examined. Bip, the major ER chaperone, acts to regulate the activation of UPR transducers. Enhanced expression of Bip has been widely used as an UPR marker (Ito et al., 2004; Lee, 2005). Western blot analysis showed that Bip was up- regulated in CHEC cells (Fig. 4A, lane 4; Fig. 4B) by an approximate 3- to 4- fold increase compared with LCEC or LSEC cells (Fig. 4A, lane 2 and 3; Fig. 4B). IER1/XBP1 is an important branch of the UPR. Its activation was studied by monitoring the splicing of $26 \mathrm{bp}$ fragment from XBP1 transcript. Amplification of the 442 bp fragment of the XBP1 cDNA was used to discriminate the spliced $(416 \mathrm{bp})\left(\mathrm{XBP} 1_{\mathrm{S}}\right)$ and unspliced $(442 \mathrm{bp})\left(\mathrm{XBP} 1_{\mathrm{U}}\right)$ forms of $\mathrm{XBP} 1$. The positive control was provided by LSEC cells treated with $2 \mathrm{mM}$ DTT for $30 \mathrm{~min}$. It has been reported that a $30-\mathrm{min}$ treatment is sufficient for mRNA induction of UPR targets (Shang and Lehrman, 2004). In the positive control (Fig. 4C, lane 1), two bands appeared, the minor and larger band corresponding to $\mathrm{XBP}_{\mathrm{U}}$, whereas the major and smaller band representing $\mathrm{XBP} 1_{\mathrm{S}}$, indicating that UPR was activated. In LCEC and LSEC cells (Fig. 4C, lane 2 and 3), we detected only the band corresponding to $\mathrm{XBP}_{\mathrm{U}}$, whereas in CHEC cells (Fig. 4C, lane 4), two bands appeared, the minor and major band corresponding to $\mathrm{XBP} 1_{\mathrm{U}}$ and $\mathrm{XBP} 1_{\mathrm{S}}$ respectively. Enhancing expression of Bip and UPR-specific splicing of XBP1 suggested that UPR was activated in CHEC cells.

When Derlin-1 was overexpressed in CHEC cells, UPR activation was checked. The expression level of Bip protein in the Derlin-1-pcDNA3-transfected CHEC cells (Fig. 4A, lane 

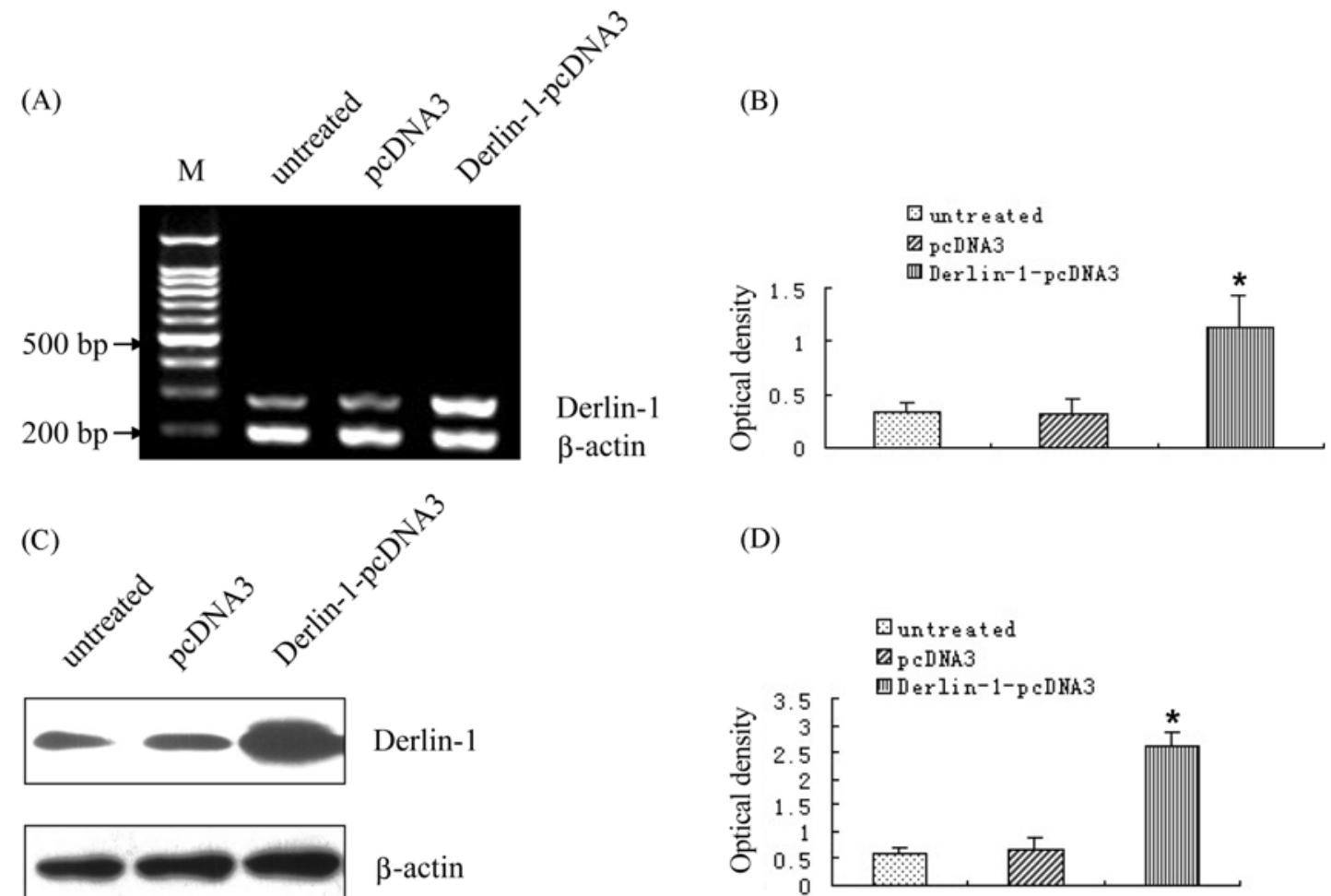

(D)

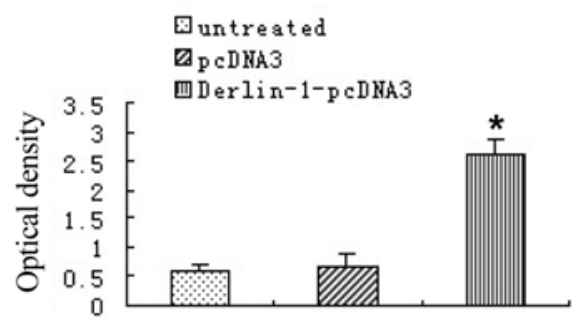

Fig. 2. Derlin-1 expression in genetically modified CHEC cells. (A) CHEC cells were transfected with pcDNA3 or Derlin-1-pcDNA3 and tested for the expression of Derlin-1 by RT-PCR. (B) The quantified Derlin-1 mRNA level in the untreated, pcDNA3-transfected, and Derlin-1-pcDNA3-transfected CHEC cells from three separated experiments. Columns, mean; bars, SE (Derlin-1-pcDNA3transfected cells versus untreated or pcDNA3-transfected control, ${ }^{*} p<0.05$ ). M, $100 \mathrm{bp}$ DNA marker. (C) Western blot analysis of Derlin-1 protein expression in the same cells as mentioned in (B). (D) Derlin-1 protein level quantitated from Western blot in (C). Columns, mean for three separate experiments; bars, SE (Derlin-1-pcDNA3-transfected cells versus untreated or pcDNA3-transfected control, $* p<0.05)$.

6; Fig. 4B) dropped to approximate $38 \%$ to $50 \%$ of the control (Fig. 4A, lane 4 and 5; Fig. 4B). With regard to XBP1, the amount of $\mathrm{XBP} 1_{\mathrm{S}}$ decreased and that of $\mathrm{XBP} 1_{\mathrm{U}}$ increased in the Derlin-1-pcDNA3-transfected cells (Fig. 4C, lane 6), as compared with the case in the empty vector-transfected and parental cells (Fig. 4C, lane 5 and 4), indicating that the XBP1 splicing was reduced. Both the reduced expression of Bip and splicing of XBP1 indicated that UPR was recovered.

\section{Discussion}

The studies presented in this report further confirmed that Derlin-1 was ubiquitously expressed in various kinds of endothelial cells, but down-regulated in CHEC cells. ER was found aberrantly enlarged in CHEC cells, but not in other tested cells. When Derlin-1 construct was introduced into CHEC cells, the dilated ER returned normal size. To explore the possible mechanism(s) responsible for this ER dynamic, UPR activation was examined. Enhanced expression of Bip and UPR-specific splicing of XBP1 were observed in CHEC cells, indicating that UPR was activated. With Derlin-1 overexpression in CHEC cells, the extent of UPR activation diminished, as manifested by decreased expression of Bip, reduced amount of $\mathrm{XBP} 1 \mathrm{~s}$, and increased expression of $\mathrm{XBP} 1_{\mathrm{U}}$.

Derlin-1 is an ER-resident transmembrane protein with both the amino and carboxy termini in the cytosol (Lilley and Ploegh, 2004). It has been suggested to play a central role in the retro-translocation of misfolded proteins from the ER to the cytosol for degradation (Lilley and Ploegh, 2004; Ye et al., 2004; Ye et al., 2005). During retro-translocation, the misfolded proteins are recognized inside the ER lumen, targeted to Derlin1, ubiquitinated and extracted from the ER membrane by Derlin-1-recruited p97 ATPase complex. Derlin-1 binds to misfolded and ubiquitinated proteins and interacts with ubiquitin ligases via binding to p97 and VIMP, thus provide the missing link between events in the ER and those in the cytosol (Ye et al., 2005; Oda et al., 2006). Derlin-1 interacts with PNGase as well, which may be required for the deglycosylation of a subset of misfolded glycoproteins, an essential process before their degradation by the proteasome (Katiyar et al., 2005). Given the role of Derlin-1 in the elimination of misfolded proteins in the ER, the ER dynamic affected by Derlin-1 overexpression prompts us to consider the possibility that ER is expanded to meet the demands for accommodating 
(A)

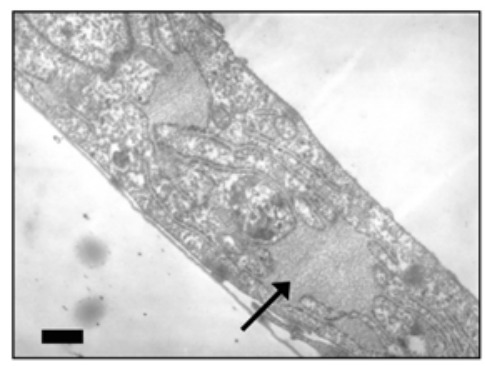

(B)

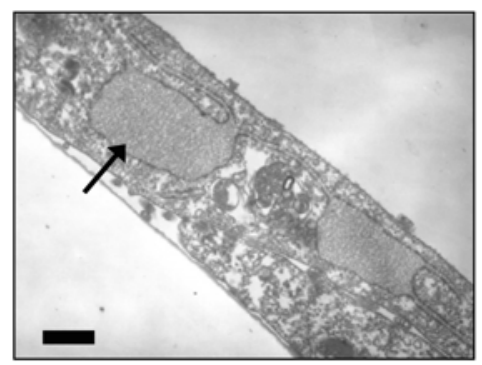

LCEC

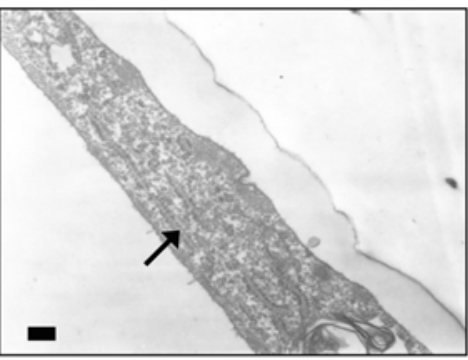

pcDNA3

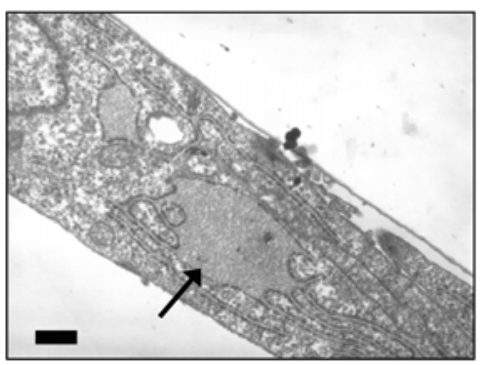

LSEC

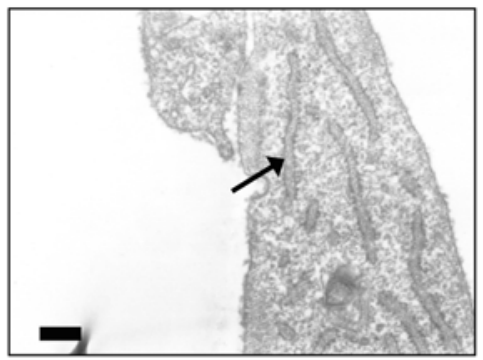

Derlin-1-pcDNA3

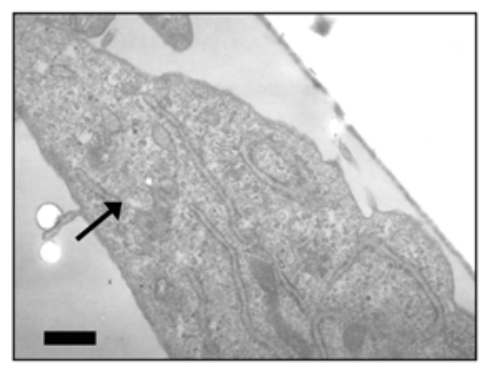

Fig. 3. Microscopy analysis of the ER. Cells were harvested, plated in cell culture inserts and cultured overnight. Then thin sections were prepared and examined by transmission electron microscopy. (A) Representative micrographs of CHEC, LCEC, and LSEC cells. (B) At $48 \mathrm{~h}$ after transfection with pcDNA3 or Derlin-1-pcDNA3, CHEC cells were subjected to microscopy analysis. Representative micrographs of three separate experiments were presented. The magnification of micrographs is shown with scale bar (200 nm) in bottom left corner. Arrows indicate representative ER. CHEC, endothelial cells derived from human hepatic hemangioma; LCEC, human liver cancer endothelial cells; LSEC, human liver sinus endothelial cells.

increased amount of misfolded proteins arising from Derlin-1 down-regulation. As described previously, ER can be expanded as well according to the demands placed upon the exocytic pathway (Sriburi et al., 2004). Basing on the above assumption, we suggest that Derlin-1 down-regulation may contribute, at least in part, to the ER expansion in CHEC cells. Indeed, derl deletion mutation had lead to considerable enlargement of the ER in yeast cells (Taxis et al., 2002).

In an effort to investigate the mechanism(s) by which overexpressed Derlin-1 inhibited ER expansion in CHEC cells, UPR, an adaptive response to cope with excessive accumulation of misfolded proteins in the ER, was examined basing on the following observations. The functions of Derlin1 homologues are well conserved from yeast to humans, and yeast derl deletion mutation induces ER expansion via UPR (Taxis et al., 2002). Similar to the case of yeast Der1, inactivation of C.elegans Derlin-1 by RNA interference can elicit UPR as well (Ye et al., 2004). Interestingly, UPR is frequently accompanied by ER expansion in mammalian cells. It has been demonstrated that IRE1-XBP1, one branch of the UPR, is required for ER proliferation in the immunoglobin secreting plasma cells (Shen et al., 2004; Sriburi et al., 2004). Similarly, simulating UPR by enforced expression of XBP1s induced phosphatidylcholine (PtdCho) biosynthesis and triggered ER expansion in fibroblasts (Sriburi et al., 2004). Recently, ER dilatation induced by tumor necrosis factor (TNF) $\alpha$ in a reactive oxygen species (ROS)-dependent fashion was observed in the murine fibrosarcoma L929 cells (Xue et al., 2005).

Bip has been well characterized as an UPR marker (Ito et al., 2004; Lee, 2005). In our study, Bip was significantly upregulated in CHEC cells, as compared with that in other tested cells, indicating that UPR was activated. One can speculate that the enhanced expression of Bip in CHEC cells may result from the different cell lines used. However, the possibility can be at least partially, if not all, ruled out, as the expression of Bip dropped after introduction of Derlin-1-pcDNA3. In fact, UPR-specific splicing of XBP1 also occurred in CHEC cells. Thus, UPR activation in CHEC cells was further confirmed. Given the role of UPR in ER expansion, the positive relation between the extent of UPR activation and ER size inferred from the Derlin-1 transfection suggests that ER expansion in CHEC cells may result from the UPR. Most importantly, XBP1 was also spliced, which has been demonstrated to play a critical role in ER expansion (Shaffer et al., 2004; Sriburi et al., 2004). Collectively, our study suggests that UPR may be involved in the ER dynamic affected by Derlin-1 overexpression in CHEC cells. Further studies are warranted to investigate how UPR inhibition affects the ER dynamic.

It is growing clear that UPR closely associates with a wide range of diseases, such as carcinogenesis (Ma and Hendershot 2004), diabetes, ischemia, and neurodegenerative disorders, 
(A)
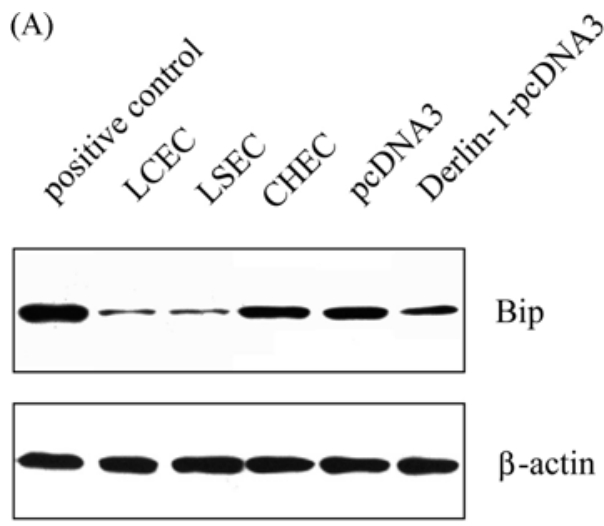

Bip

$\beta$-actin
(B)

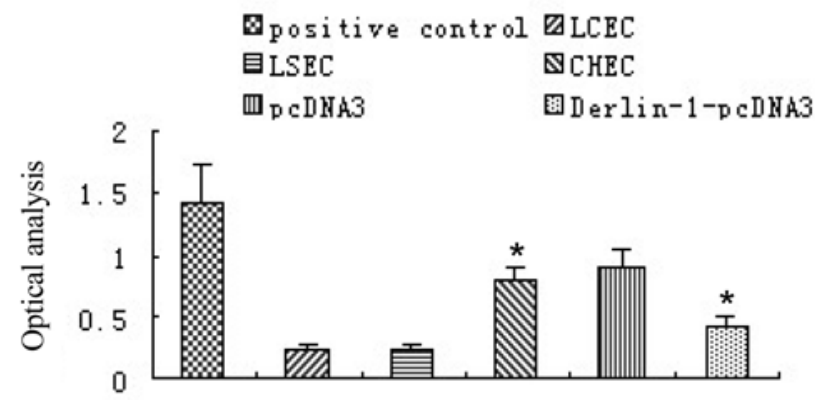

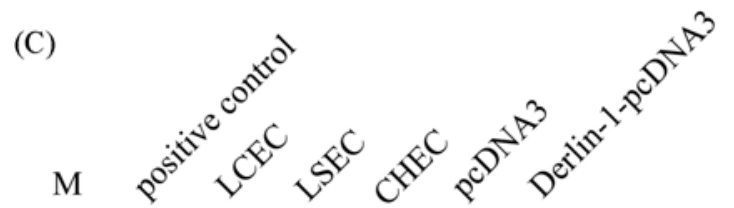

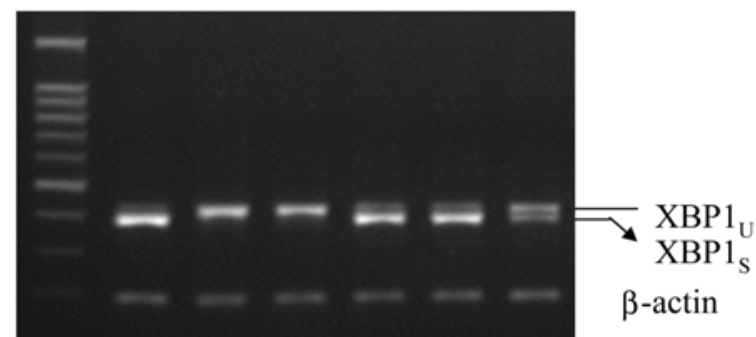

Fig. 4. Western blot analysis for Bip expression and RT-PCR analysis for XBP1 splicing. (A) Bip expression in the indicated endothelial cells revealed by Western blot analysis. (B) Bip protein level quantitated from Western blot in (A). Columns, mean for three separate experiments; bars, SE (CHEC cells versus LCEC or LSEC cells, ${ }^{*} p<0.05$; Derlin-1-pcDNA3-transfected CHEC cells versus untreated or pcDNA3-transfected control, ${ }^{*} p<0.05$ ). (C) XBP1 splicing in the indicated cells revealed by RT-PCR analysis. Three separate experiments were performed. M, 100 bp DNA marker; LCEC, human liver cancer endothelial cells; LSEC, human liver sinus endothelial cells; CHEC, endothelial cells derived from human hepatic hemangioma; positive control, LSEC cells treated with $2 \mathrm{mM}$ DTT for $30 \mathrm{~min}$; XBP1 $1_{\mathrm{U}}$, unspliced form of XBP1; XBP1s, spliced form of XBP1.

making ER a possible instigator of pathological cell death and dysfunction (Xu et al., 2005). Hepatic cavernous hemangioma is the most frequent benign liver tumor. However, its pathogenesis remains poorly understood. Recently, genetic defects have been implicated in the pathogenesis of cerebral and retinal cavernous angiomas (Couteulx et al., 2002; Plummer et al., 2005; Zawistowski et al., 2005). Future studies are warranted to investigate the role of Derlin-1 downregulation and ER expansion in the pathogenesis of hepatic cavernous hemangioma and determine whether the UPR associates with it.

In conclusion, we demonstrate that overexpressed Derlin-1 inhibits ER expansion in CHEC cells where Derlin-1 is markedly down-regulated. Furthermore, we strongly suggest that UPR may be responsible for the ER dynamic affected by Derlin-1 overexpression. Thus, we provide another example of a single protein being able to affect ER dynamic in mammalian cells, and an insight into the possible molecular mechanism(s).
Acknowledgments We thank Dr. Yangfu Jiang for critical reading of this manuscript. This work was supported by National Natural Science Foundation of China (Key Program) (No. 30230150) and National High Technology Research and Development Program of China (863 Program) (No. 2001AA221251).

\section{References}

Couteulx, S. L., Brezin A. P., Fontaine, B., Tournier-Lasserve, E. and Labauge, P. (2002) A novel KRIT1/CCM1 truncating mutation in a patient with cerebral and retinal cavernous angiomas. Arch. Ophthalmol. 120, 217-218.

Dalby, B., Cates, S., Harris, A., Ohki, E. C., Tilkins, M. L., Price, P. J. and Ciccarone, V. C. (2004) Advanced transfection with Lipofectamine 2000 reagent: primary neurons, siRNA, and high-throughput applications. Methods 33, 95-103.

Federovitch, C. M., Ron, D. and Hampton, R. Y. (2005) The dynamic ER: experimental approaches and current questions. 
Curr. Opin. Cell Biol. 17, 409-414.

Hosokawa, N., Wada, I., Hasegawa, K., Yorihuzi, T., Tremblay, L. O., Herscovics, A. and Nagata, K. (2001) A novel ER alphamannosidase-like protein accelerates ER-associated degradation. EMBO Rep. 2, 415-422.

Hough, R. F., Lingam, A. T. and Bass, B. L. (1999) Caenorhabditis elegans mRNAs that encode a protein similar to ADARs derive from an operon containing six genes. Nucleic Acids Res. 27, 3424-3432.

Ito, D., Walker, J. R., Thompson, C. S., Moroz, I., Lin, W., Veselits, M. L., Hakim, A. M., Fienberg, A. A. and Thinakaran, G. (2004) Characterization of stanniocalcin 2, a novel target of the mammalian unfolded protein response with cytoprotective properties. Mol. Cell Biol. 24, 9456-9469.

Jaffe, E. A., Nachman, R. L., Becker, C. G. and Minick, C. R. (1973) Culture of human endothelial cells derived from umbilical veins. Identification by morphologic and immunologic criteria. J. Clin. Invest. 52, 2745-2756.

Kalies, K. U., Allan, S., Sergeyenko, T., Kroger, H. and Romisch, K. (2005) The protein translocation channel binds proteasomes to the endoplasmic reticulum membrane. $E M B O J . \mathbf{2 4}, 2284-$ 2293.

Katiyar, S., Joshi, S. and Lennarz, W. J. (2005) The retrotranslocation protein derlin-1 binds peptide:N-glycanase to the endoplasmic reticulum. Mol. Biol. Cell 16, 4584-4594.

Kirst, M. E., Meyer, D. J., Gibbon, B. C., Jung, R. and Boston, R. S. (2005) Identification and characterization of endoplasmic reticulum-associated degradation proteins differentially affected by endoplasmic reticulum stress. Plant Physiol. 138, 218-231.

Knop, M., Finger, A., Braun, T., Hellmuth, K. and Wolf, D. H. (1996) Der1, a novel protein specifically required for endoplasmic reticulum degradation in yeast. EMBO $J$ 15, 753-763.

Lee, A. S. (2005) The ER chaperone and signaling regulator GRP78/BiP as a monitor of endoplasmic reticulum stress. Methods 35, 373-381.

Lilley, B. N. and Ploegh H. L. (2004) A membrane protein required for dislocation of misfolded proteins from the ER. Nature 429, 834-840.

Lilley, B. N. and Ploegh, H. L. (2005) Multiprotein complexes that link dislocation, ubiquitination, and extraction of misfolded proteins from the endoplasmic reticulum membrane. Proc. Natl. Acad. Sci. USA 102, 14296-14301.

Lou, J., Buhler, L., Deng, S., Mentha, G., Montesano, R., Grau, G. E. and Morel, P. (1998) Inhibition of leukocyte adherence and transendothelial migration in cultured human liver vascular endothelial cells by prostaglandin E1. Hepatology 27, 822-828.

Lou, J., Triponez, F., Oberholzer, J., Wang, H., Yu, D., Buhler, L., Cretin, N., Mentha, G., Wollheim, C. B. and Morel, P. (1999) Expression of alpha-1 proteinase inhibitor in human islet microvascular endothelial cells. Diabetes 48, 1773-1778.

Ma, Y. and Hendershot, L. M. (2004) Herp is dually regulated by both the endoplasmic reticulum stress-specific branch of the unfolded protein response and a branch that is shared with other cellular stress pathways. J. Biol. Chem. 279, 1379213799.

Ma, Y. and Hendershot, L. M. (2004) The role of the unfolded protein response in tumour development: friend or foe? Nat. Rev. Cancer 4, 966-977.

Mori, K. (2003) Frame switch splicing and regulated intramembrane proteolysis: key words to understand the unfolded protein response. Traffic 4, 519-28.

Oda, Y., Okada, T., Yoshida, H., Kaufman, R. J., Nagata, K. and Mori, K. (2006) Derlin-2 and Derlin-3 are regulated by the mammalian unfolded protein response and are required for ERassociated degradation. J. Cell Biol. 172, 383-193.

Plummer, N. W., Zawistowski, J. S. and Marchuk, D. A. (2005) Genetics of cerebral cavernous malformations. Curr. Neurol. Neurosci. Rep. 5, 391-396.

Salmon, P., Oberholzer, J., Occhiodoro, T., Morel, P., Lou, J. and Trono, D. (2000) Reversible immortalization of human primary cells by lentivector-mediated transfer of specific genes. Mol. Ther. 2, 404-414.

Schroder, M. and Kaufman, R. J. (2005) The mammalian unfolded protein response. Annu. Rev. Biochem. 74, 739-789.

Shaffer, A. L., Shapiro-Shelef, M., Iwakoshi, N. N., Lee, A. H., Qian, S. B., Zhao, H., Yu, X., Yang, L., Tan, B. K., Rosenwald, A., Hurt, E. M., Petroulakis, E., Sonenberg, N., Yewdell, J. W., Calame, K., Glimcher, L. H. and Staudt, L. M. (2004) XBP1, downstream of Blimp-1, expands the secretory apparatus and other organelles, and increases protein synthesis in plasma cell differentiation. Immunity 21, 81-93.

Shang, J. and Lehrman, M. A. (2004) Discordance of UPR signaling by ATF6 and Ire1p-XBP1 with levels of target transcripts. Biochem. Biophys. Res. Commun. 317, 390-396.

Shen, X., Zhang, K. and Kaufman, R. J. (2004) The unfolded protein response--a stress signaling pathway of the endoplasmic reticulum. J. Chem. Neuroanat. 28, 79-92.

Sriburi, R., Jackowski, S., Mori, K. and Brewer, J. W. (2004) XBP1: a link between the unfolded protein response, lipid biosynthesis, and biogenesis of the endoplasmic reticulum. $J$. Cell Biol. 167, 35-41.

Taxis, C., Vogel, F. and Wolf, D. H. (2002) ER-golgi traffic is a prerequisite for efficient ER degradation. Mol. Biol. Cell 13, 1806-1818.

Travers, K. J., Patil, C. K., Wodicka, L., Lockhart, D. J., Weissman, J. S. and Walter, P. (2000) Functional and genomic analyses reveal an essential coordination between the unfolded protein response and ER-associated degradation. Cell 101, 249258.

Tsai, B., Ye, Y. and Rapoport, T. A. (2002) Retro-translocation of proteins from the endoplasmic reticulum into the cytosol. Nat. Rev. Mol. Cell Biol. 3, 246-255.

Xu, C., Bailly-Maitre, B. and Reed, J. C. (2005) Endoplasmic reticulum stress: cell life and death decisions. J. Clin. Invest. 115, 2656-2664.

Xue, X., Piao, J. H., Nakajima, A., Sakon-Komazawa, S., Kojima, Y., Mori, K., Yagita, H., Okumura, K., Harding, H. and Nakano, H. (2005) Tumor Necrosis Factor $\alpha$ (TNF $\alpha$ ) Induces the Unfolded Protein Response (UPR) in a Reactive Oxygen Species (ROS)-dependent Fashion, and the UPR Counteracts ROS Accumulation by TNF $\alpha$. J. Biol. Chem. 280, 3391733925.

Yamamoto, K., Yoshida, H., Kokame, K., Kaufman, R. J. and Mori, K. (2004) Differential contributions of ATF6 and XBP1 to the activation of endoplasmic reticulum stress-responsive cisacting elements ERSE, UPRE and ERSE-II. J. Biochem. (Tokyo) 136, 343-350.

Ye, Y., Shibata, Y., Kikkert, M., van Voorden, S., Wiertz, E. and Rapoport, T. A. (2005) Recruitment of the p97 ATPase and ubiquitin ligases to the site of retrotranslocation at the 
endoplasmic reticulum membrane. Proc. Natl. Acad. Sci. USA 102, 14132-14138.

Ye, Y., Shibata, Y., Yun, C., Ron, D. and Rapoport, T. A. (2004) A membrane protein complex mediates retro-translocation from the ER lumen into the cytosol. Nature 429, 841-847.

Zawistowski, J. S., Stalheim, L., Uhlik, M. T., Abell, A. N., Ancrile, B. B., Johnson, G. L. and Marchuk, D. A. (2005) CCM1 and CCM2 protein interactions in cell signaling: implications for cerebral cavernous malformations pathogenesis. Hum. Mol. Genet. 14, 2521-2531.

Zhong, X., Ran, Y. L., Lou, J. N., Hu, D., Yu, L., Zhang, Y. S., Zhou, Z. and Yang, Z. H. (2004) Construction of human liver cancer vascular endothelium cDNA expression library and screening of the endothelium-associated antigen genes. World $J$. Gastroenterol. 10, 1402-1408. 